\title{
Key-Value Retrieval Networks for Task-Oriented Dialogue
}

\author{
Mihail Eric $^{1}$, Lakshmi Krishnan ${ }^{2}$, \\ Francois Charette $^{2}$, and Christopher D. Manning ${ }^{1}$ \\ mericlcs.stanford.edu, lkrishn7@ford.com \\ fcharett@ford. com, manning@stanford.edu \\ Stanford NLP Group ${ }^{1}$ Ford Research and Innovation Center ${ }^{2}$
}

\begin{abstract}
Neural task-oriented dialogue systems often struggle to smoothly interface with a knowledge base. In this work, we seek to address this problem by proposing a new neural dialogue agent that is able to effectively sustain grounded, multi-domain discourse through a novel key-value retrieval mechanism. The model is end-to-end differentiable and does not need to explicitly model dialogue state or belief trackers. We also release a new dataset of 3,031 dialogues that are grounded through underlying knowledge bases and span three distinct tasks in the in-car personal assistant space: calendar scheduling, weather information retrieval, and point-of-interest navigation. Our architecture is simultaneously trained on data from all domains and significantly outperforms a competitive rulebased system and other existing neural dialogue architectures on the provided domains according to both automatic and human evaluation metrics.
\end{abstract}

\section{Introduction}

With the success of new speech-based humancomputer interfaces, there is a great need for effective task-oriented dialogue agents that can handle everyday tasks such as scheduling events and booking hotels. Current commercial dialogue agents are often brittle pattern-matching systems which are unable to maintain the kind of flexible conversations that people desire. Neural dialogue agents present one of the most promising avenues for leveraging dialogue corpora to build statistical models directly from data by using powerful distributed representations (Bordes and Weston, 2016; Wen et al., 2016b; Dhingra et al., 2016).

\begin{tabular}{|c|c|c|c|c|}
\hline Event & Time & Date & Party & Agenda \\
\hline opt. appt. & $10 \mathrm{am}$ & Thursday & sister & - \\
dinner & $8 \mathrm{pm}$ & the 13th & Ana & - \\
opt. appt. & $7 \mathrm{pm}$ & the 20 th & Jeff & - \\
opt. appt. & $4 \mathrm{pm}$ & the 13 th & Alex & - \\
$\ldots$ & $\ldots$ & $\ldots$ & $\ldots$ & $\ldots$ \\
\hline
\end{tabular}

DRIVER: I need to find out the time and parties attending my optometrist appointment.

CAR: I have 3 appointments scheduled, with Alex, your sister, and Jeff. Which are you referring to?

DRIVER: I want to know about the one that Alex is

$\begin{array}{ll} & \text { joining me at } \\ \text { CAR: } & \text { That optometrist appointment is at } 4 \mathrm{pm} \text {. }\end{array}$

DRIVER: Thanks

CAR: no problem

Figure 1: Sample dialogue from our dataset. Note some columns and rows from the knowledge base are not included due to space constraints. A dash indicates a missing value.

While this work has been somewhat successful, these task-oriented neural dialogue models suffer from a number of problems: 1) They struggle to effectively reason over and incorporate knowledge base information while still preserving their endto-end trainability and 2) They often require explicitly modelling user dialogues with belief trackers and dialogue state information, which necessitates additional data annotation and also breaks differentiability.

To address some of the modelling issues in previous neural dialogue agents, we introduce a new architecture called the Key-Value Retrieval Network. This model augments existing recurrent network architectures with an attention-based key-value retrieval mechanism over the entries of a knowledge base, which is inspired by recent work on key-value memory networks (Miller et al., 2016). By doing so, it is able to learn how to extract useful information from a knowledge base directly from data in an end-to-end fashion, with- 
out the need for explicit training of belief or intent trackers as is done in traditional task-oriented dialogue systems. The architecture has no dependence on the specifics of the data domain, learning how to appropriately incorporate world knowledge into its dialogue utterances via attention over the key-value entries of the underlying knowledge base.

In addition, we introduce and make publicly available a new corpus of 3,031 dialogues spanning three different domain types in the incar personal assistant space: calendar scheduling, weather information retrieval, and point-ofinterest navigation. The dialogues are grounded through knowledge bases. This makes them ideal for building dialogue architectures that seamlessly reason over world knowledge. The multi-domain nature of the dialogues in the corpus also makes this dataset an apt test bed for generalizability of modelling architectures. ${ }^{1}$

The main contributions of our work are therefore two-fold: 1) We introduce the Key-Value Retrieval Network, a highly performant neural taskoriented dialogue agent that is able to smoothly incorporate information from underlying knowledge bases through a novel key-value retrieval mechanism. Unlike other dialogue agents which only rely on prior dialogue history for generation (Kannan et al., 2016; Eric and Manning, 2017), our architecture is able to access and use database-style information, while still retaining the text generation advantages of recent neural models. By doing so, our model outperforms a competitive rulebased system and other baseline neural models on a number of automatic metrics as well as human evaluation. 2) We release a new publicly-available dialogue corpus across three distinct domains in the in-car personal assistant space that we hope will help further work on task-oriented dialogue agents.

\section{Key-Value Retrieval Networks}

While recent neural dialogue models have explicitly modelled dialogue state through belief and user intent trackers (Wen et al., 2016b; Dhingra et al., 2016; Henderson et al., 2014b), we choose instead to rely on learned neural representations for implicit modelling of dialogue state, forming

\footnotetext{
${ }^{1}$ The data is available for download at https://nlp.stanford.edu/blog/a-new-multi-turn-multidomain-task-oriented-dialogue-dataset/
}

a truly end-to-end trainable system. Our model starts with an encoder-decoder sequence architecture and is further augmented with an attentionbased retrieval mechanism that effectively reasons over a key-value representation of the underlying knowledge base. We describe each component of our model in the subsequent sections.

\subsection{Encoder}

Given a dialogue between a user $(u)$ and a system $(s)$, we represent the dialogue utterances as $\left\{\left(u_{1}, s_{1}\right),\left(u_{2}, s_{2}\right), \ldots,\left(u_{k}, s_{k}\right)\right\}$ where $k$ denotes the number of turns in the dialogue. At the $i^{\text {th }}$ turn of the dialogue, we encode the aggregated dialogue context composed of the tokens of $\left(u_{1}, s_{1}, \ldots, s_{i-1}, u_{i}\right)$. Letting $x_{1}, \ldots, x_{m}$ denote these tokens, we first embed these tokens using a trained embedding function $\phi^{e m b}$ that maps each token to a fixed-dimensional vector. These mappings are fed into the encoder to produce contextsensitive hidden representations $h_{1}, \ldots, h_{m}$, by repeatedly applying the recurrence:

$$
h_{i}=\operatorname{LSTM}\left(\phi^{e m b}\left(x_{i}\right), h_{i-1}\right)
$$

where the recurrence uses a long-short-term memory unit, as described by (Hochreiter and Schmidhuber, 1997).

\subsection{Decoder}

The vanilla sequence-to-sequence decoder predicts the tokens of the $i^{\text {th }}$ system response $s_{i}$ by first computing decoder hidden states via the recurrent unit. We denote $\tilde{h}_{1}, \ldots, \tilde{h}_{n}$ as the hidden states of the decoder and $y_{1}, \ldots, y_{n}$ as the output tokens. We extend this decoder with an attentionbased model (Bahdanau et al., 2015; Luong et al., 2015a), where, at every time step $t$ of the decoding, an attention score $a_{i}^{t}$ is computed for each hidden state $h_{i}$ of the encoder, using the attention mechanism of (Vinyals et al., 2015). Formally this attention can be described by the following equations:

$$
\begin{aligned}
u_{i}^{t} & \left.=w^{T} \tanh \left(W_{2} \tanh \left(W_{1}\left[h_{i}, \tilde{h}_{t}\right]\right)\right)\right) \\
a_{i}^{t} & =\operatorname{Softmax}\left(u_{i}^{t}\right) \\
\tilde{h}_{t}^{\prime} & =\sum_{i=1}^{m} a_{i}^{t} h_{i} \\
o_{t} & =U\left[\tilde{h}_{t}, \tilde{h}_{t}^{\prime}\right] \\
y_{t} & =\operatorname{Softmax}\left(o_{t}\right)
\end{aligned}
$$


where $U, W_{1}, W_{2}$, and $w$ are trainable parameters of the model and $o_{t}$ represents the logits over the tokens of the output vocabulary $V$. In (2) above, the attention logit on $h_{i}$ is computed via a twolayer MLP function with a tanh nonlinearity at the intermediate layers. During training, the next token $y_{t}$ is predicted so as to maximize the loglikelihood of the correct output sequence given the input sequence.

\subsection{Key-Value Knowledge Base Retrieval}

Recently, some neural task-oriented dialogue agents that query underlying knowledge bases (KBs) and extract relevant entities either do the following: 1) create and execute well-formatted API calls to the KB, operations which require intermediate supervision in the form of training slot trackers and which break differentiability (Wen et al., 2016b), or 2) softly attend to the KB and combine this probability distribution with belief trackers as state input for a reinforcement learning policy (Dhingra et al., 2016). We choose to build off the latter approach as it fits nicely into the end-to-end trainable framework of sequenceto-sequence modelling, though we are in a supervised learning setting and we do away with explicit representations of belief trackers or dialogue state.

For storing the $\mathrm{KB}$ of a given dialogue, we take inspiration from the work of (Miller et al., 2016) which found that a key-value structured memory allowed for efficient machine reading of documents. We store every entry of our KB using a (subject, relation, object) representation. In our representation a KB entry from the dialogue in Figure 1 such as (event $=$ dinner, time $=8 p m$, date=the 13th, party=Ana, agenda="-") would be normalized into four separate triples of the form (dinner, time, 8pm). Every $\mathrm{KB}$ has at most 230 normalized triples. This formalism is similar to a neo-Davidsonian or RDF-style representation of events.

Recent literature has shown that incorporating a copying mechanism into neural architectures improves performance on various sequenceto-sequence tasks (Jia and Liang, 2016; Gu et al., 2016; Ling et al., 2016; Gulcehre et al., 2016; Eric and Manning, 2017). We build off this intuition in the following way: at every timestep of decoding, we take the decoder hidden state and compute an attention score with the key of each normalized
KB entry. For our purposes, the key of an entry corresponds to the sum of the word embeddings of the subject (meeting) and relation (time). The attention logits then become the logits of the value for that KB entry. For our KB attentions, we replace the embedding of the value with a canonicalized token representation. For example, the value $5 \mathrm{pm}$ is replaced with the canonicalized representation meeting_time. At runtime, if we decode this canonicalized representation token, we convert it into the actual value of the $\mathrm{KB}$ entry $(5 \mathrm{pm}$ in our running example) through a KB lookup. Note that this means we are expanding our original output vocabulary to $|V|+n$ where $n$ is the number of separate canonical key representation KB entries.

In particular, let $k_{j}$ denote the word embedding of the key of our $j^{\text {th }}$ normalized KB entry. We can now formalize the decoding for our KB attentionbased retrieval. Assume that we have $m$ distinct triples in our $\mathrm{KB}$ and that we are in the $t^{\text {th }}$ timestep of decoding:

$$
\begin{aligned}
u_{j}^{t} & \left.=r^{T} \tanh \left(W_{2}^{\prime} \tanh \left(W_{1}^{\prime}\left[k_{j}, \tilde{h}_{t}\right]\right)\right)\right) \\
o_{t} & =U\left[\tilde{h}_{t}, \tilde{h}_{t}^{\prime}\right]+\bar{v}^{t} \\
y_{t} & =\operatorname{Softmax}\left(o_{t}\right)
\end{aligned}
$$

where $r, W_{1}^{\prime}$, and $W_{2}^{\prime}$ are trainable parameters. In (8) above, $\bar{v}^{t}$ is a sparse vector with length $|V|+n$. Within $\bar{v}^{t}$, the entry for the value embedding $v_{j}$ corresponding to the key $k_{j}$ is equal to the logit score $u_{j}^{t}$ on $k_{j}$. Hence, the $m$ entries of $\bar{v}^{t}$ corresponding to the values in the $\mathrm{KB}$ are non-zero, whereas the remaining entries corresponding to the original vocabulary tokens are 0 . This sparse vector contains our aggregated KB logit scores which we combine with the original logits to get a modified $o_{t}$. We then select the argmax token as input to the next timestep. This description seeks to capture the intuition that in response to the query What time is my meeting, we want the model to put a high attention weight on the key representation for the (meeting, time, $5 \mathrm{pm}$ ) KB triple, which should then lead the model to favor outputting the value token at the given timestep. We provide a visualization of the KeyValue Retrieval Network in Figure 2.

\section{A Multi-Turn, Multi-Domain Dialogue Dataset}

In an effort to further work in multi-domain dialogue agents, we built a corpus of multi-turn 


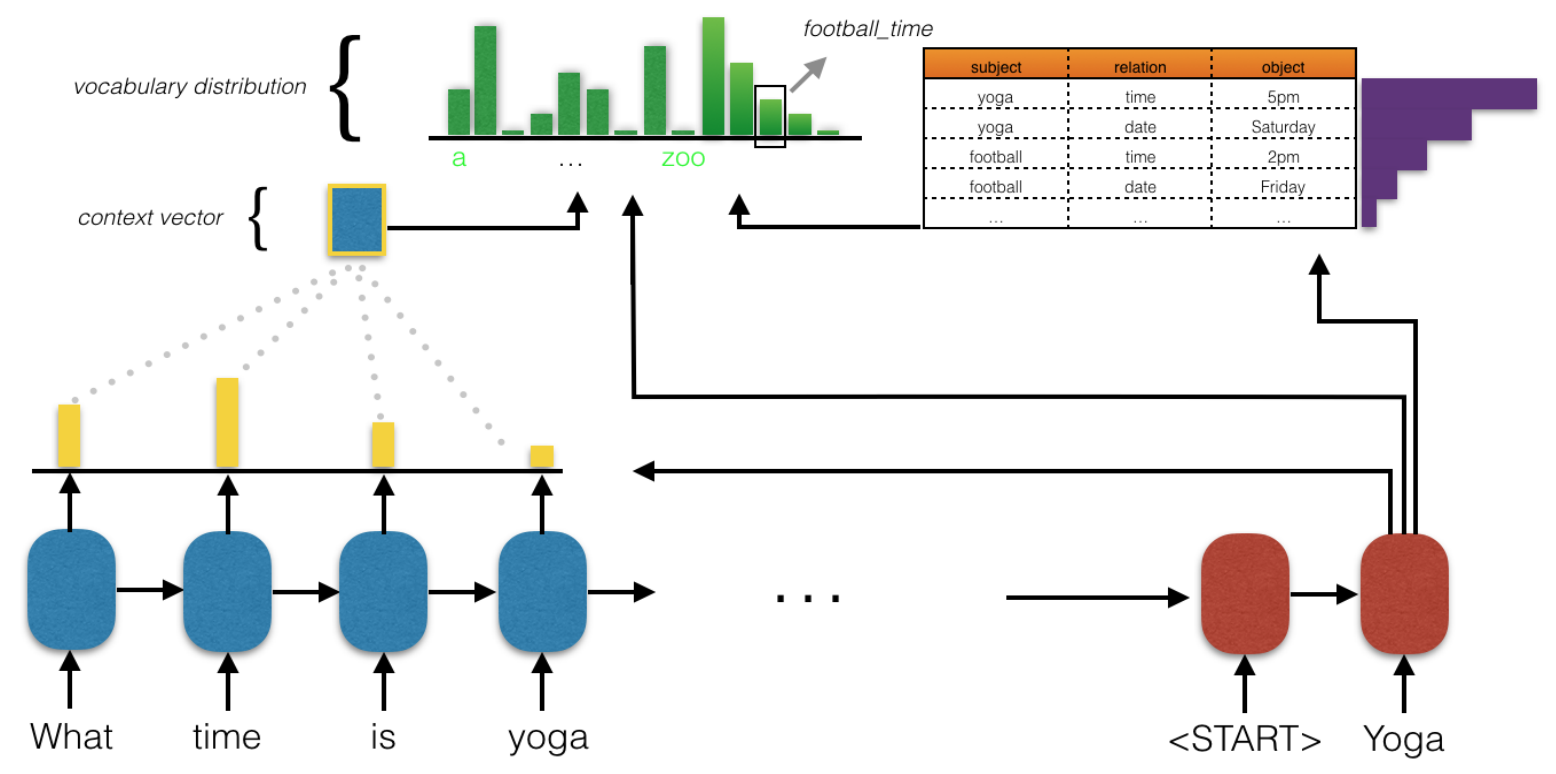

Figure 2: Key-value retrieval network. For each time-step of decoding, the cell state is used to compute an attention over the encoder states and a separate attention over the key of each entry in the KB. The attentions over the encoder are used to generate a context vector which is combined with the cell state to get a distribution over the normal vocabulary. The attentions over the keys of the KB become the logits for their associated values and are separate entries in a now augmented vocabulary that we argmax over.

dialogues in three distinct domains: calendar scheduling, weather information retrieval, and point-of-interest navigation. While these domains are different, they are all relevant to the overarching theme of tasks that users would expect of a sophisticated in-car personal assistant.

\subsection{Data Collection}

The data for the multi-turn dialogues was collected using a Wizard-of-Oz scheme inspired by that of (Wen et al., 2016b). In our scheme, users had two potential modes they could play: Driver and Car Assistant. In the Driver mode, users were presented with a task that listed certain information they were trying to extract from the Car Assistant as well as the dialogue history exchanged between Driver and Car Assistant up to that point. An example task presented could be: You want to find what the temperature is like in San Mateo over the next two days. The Driver was then only responsible for contributing a single line of dialogue that appropriately continued the discourse given the prior dialogue history and the task definition.

Tasks were randomly specified by selecting values (5pm, Saturday, San Francisco, etc.) for three to five slots (time, date, location, etc.), de- pending on the domain type. Values specified for the slots were chosen according to a uniform distribution from a per-domain candidate set.

In the Car Assistant mode, users were presented with the dialogue history exchanged up to that point in the running dialogue and a private knowledge base known only to the Car Assistant with information that could be useful for satisfying the Driver query. Examples of knowledge bases could include a calendar of event information, a collection of weekly forecasts for nearby cities, or a collection of nearby points-of-interest with relevant information. The Car Assistant was then responsible for using this private information to provide a single utterance that progressed the user-directed dialogues. The Car Assistant was also asked to fill in dialogue state information for mentioned slots and values in the dialogue history up to that point.

Each private knowledge base had six to seven distinct rows and five to seven attribute types. The private knowledge bases used were generated by uniformly selecting a value for a given attribute type, where each attribute type had a variable number of candidate values. Some knowledge bases intentionally lacked attributes to encourage diversity in discourse.

During data collection, some of the dialogues 


\begin{tabular}{l|c|c|c} 
& Calendar Scheduling & Weather Information Retrieval & POI Navigation \\
\hline Slot Types & $\begin{array}{c}\text { event, time, date, } \\
\text { party, room, agenda }\end{array}$ & $\begin{array}{c}\text { location, weekly time, } \\
\text { temperature, weather attribute }\end{array}$ & $\begin{array}{c}\text { POI name, traffic info, } \\
\text { POI category, address, distance }\end{array}$ \\
\hline \# Distinct Slot Values & 79 & 65 & 140 \\
\hline
\end{tabular}

Table 1: Slots types and number distinct slot values for different domains. POI denotes point-of-interest.

\begin{tabular}{|l|r|}
\hline Training Dialogues & 2,425 \\
Validation Dialogues & 302 \\
Test Dialogues & 304 \\
Calendar Scheduling Dialogues & 1034 \\
Navigation Dialogues & 1000 \\
Weather Dialogues & 997 \\
Avg. \# of Utterances Per Dialogue & 5.25 \\
Avg. \# of Tokens Per Utterance & 9 \\
Vocabulary Size & 1,601 \\
\# of Distinct Entities & 284 \\
\# of Entity (or Slot) Types & 15 \\
\hline
\end{tabular}

Table 2: Statistics of Dataset.

in the calendar scheduling domain did not explicitly require the use of a KB. For example, in a task such as Set a meeting reminder at 3pm, we hoped to encourage dialogues that required the Car Assistant to execute a task while asking for Driver clarification on underspecified information. Roughly half of the scheduling dialogues fell into this category.

While specifying the attribute types and values in each task presented to the Driver allowed us to ground the subject of each dialogue with our desired entities, it would occasionally result in more mechanical discourse exchanges. To encourage more naturalistic, unbiased utterances, we had users record themselves saying commands in response to underspecified visual depictions of an action a car assistant could perform. These commands were transcribed and then inserted as the first exchange in a given dialogue on behalf of the Driver. Roughly $\sim 1,500$ of the dialogues employed this transcribed audio command firstutterance technique.

241 unique workers from Amazon Mechanical Turk were anonymously recruited to use the interface we built over a period of about six days. Data statistics are provided in Table 1 and slot types and values are provided in Table 2. A screenshot of the user-facing interfaces for the data collection, as well as a visual used to prompt user recorded commands, are provided in the supplementary material.

\section{Related Work}

Task-oriented agents for spoken dialogue systems have been the subject of extensive research effort. One line of work by (Young et al., 2013) has tackled the problem using partially observable Markov decision processes and reinforcement learning with carefully designed action spaces, though the number of distinct action states makes this approach often brittle and computationally intractable.

The recent successes of neural architectures on a number of traditional natural language processing subtasks (Bahdanau et al., 2015; Sutskever et al., 2014; Vinyals et al., 2015) have motivated investigation into dialogue agents that can effectively make use of distributed neural representations for dialogue state management, belief tracking, and response generation. Recent work by (Wen et al., 2016b) has built systems with modularly-connected representation, belief state, and generation components. These models learn to explicitly represent user intent through intermediate supervision, which breaks end-to-end trainability. Other work by (Bordes and Weston, 2016; Liu and Perez, 2016) stores dialogue context in a memory module and repeatedly queries and reasons about this context to select an adequate system response from a set of all candidate responses.

Another line of recent work has developed taskoriented models which are amenable to both supervised learning and reinforcement learning and are able to incorporate domain-specific knowledge via explicitly-provided features and model-output restrictions (Williams et al., 2017). Our model contrasts with these works in that training is done in a strictly supervised fashion via a per utterance token generative process, and the model does not need dialogue state trackers, relying instead on latent neural embeddings for accurate system response generation.

Research in task-oriented dialogue often struggles with a lack of standard, publicly available datasets. Several classical corpora have consisted of moderately-sized collections of dialogues related to travel-booking (Hemphill et al., 1990; 
Bennett and Rudnicky, 2002). Another wellknown corpus is derived from a series of competitions on the task of dialogue-state tracking (Williams et al., 2013). While the competitions were designed to test systems for state tracking, recent work has chosen to repurpose this data by only using the transcripts of dialogues without state annotation for developing systems (Bordes and Weston, 2016; Williams et al., 2017). More recently, Maluuba has released a dataset of hotel and travel-booking dialogues collected in a Wizard-ofOz Scheme with elaborate semantic frames annotated (Asri et al., 2017). This dataset aims to encourage research in non-linear decision-making processes that are present in task-oriented dialogues.

\section{Experiments}

In this section we first introduce the details of the experiments and then present results from both automatic and human evaluation.

\subsection{Details}

For our experiments, we divided the dialogues into train/validation/test sets using a 0.8/0.1/0.1 data split and ensured that each domain type was equally represented in each of the splits.

To reduce lexical variability, in a pre-processing step, we map the variant surface expression of entities to a canonical form using named entity recognition and linking. For example, the surface form 20 Main Street is mapped to Pizza My Heart address. During inference, our model outputs the canonical forms of the entities, and so we realize their surface forms by running the system output through an inverse lexicon. The inverse lexicon converts the entities back to their surface forms by sampling from a multinomial distribution with parameters of the distribution equal to the frequency count of a given surface form for an entity as observed in the training and validation data. Note that for the purposes of computing our evaluation metrics, we operate on the canonicalized forms, so that any non-deterministic variability in surface form realization does not affect the computed metrics.

\subsection{Hyperparameters}

We trained using a cross-entropy loss and the Adam optimizer (Kingma and Ba, 2015) with learning rates sampled from the interval $\left[10^{-4}, 10^{-3}\right]$. We applied dropout (Hinton et al., 2012) as a regularizer to the input and output of the LSTM. We also added an $l_{2}$ regularization penalty on the weights of the model. We identified hyperparameters by random search, evaluating on the held-out validation subset of the data. Dropout keep rates were sampled from $[0.8,0.9]$ and the $l_{2}$ coefficient was sampled from $\left[3 \cdot 10^{-6}, 10^{-5}\right]$. We used word embeddings, hidden layer, and cell sizes with size 200. We applied gradient clipping with a clip-value of 10 to avoid gradient explosions during training. The attention, output parameters, word embeddings, and LSTM weights were randomly initialized from a uniform unit-scaled distribution in the style of (Sussillo and Abbott, 2015). We also added a bias of 1 to the LSTM cell forget gate in the style of (Pham et al., 2014).

\subsection{Baseline Models}

We provide several baseline models for comparing performance of the Key-Value Retrieval Network:

- Rule-Based Model: This model is a traditional rule-based system with modular dialogue state trackers, KB query, and natural language generation components. It first does an extensive domain-dependent keyword search in the user utterances to detect intent. The user utterances are also provided to a lexicon to extract any entities mentioned. Collectively, this information forms the dialogue state up to a given point in the dialogue. This dialogue state is used to query the KB as appropriate, and the returned $\mathrm{KB}$ values are used to fill in predefined template system responses.

\section{- Copy-Augmented Sequence-to-Sequence} Network: This model is derived from the work of (Eric and Manning, 2017). It augments a sequence-to-sequence architecture with encoder attention, with an additional attention-based hard-copy mechanism over the $\mathrm{KB}$ entities mentioned in the encoder context. This model does not explicitly incorporate information from the underlying $\mathrm{KB}$ and instead relies solely on dialogue history for system response generation. Unlike the best performing model of (Eric and Manning, 2017), we do not enhance the inputs to the encoder with additional entity type features, as we found that the 


\begin{tabular}{llcccc} 
Model & BLEU & Ent. $_{1}$ & Scheduling Ent. $\mathbf{F}_{1}$ & Weather Ent. $\mathbf{F}_{1}$ & Navigation Ent. $\mathbf{F}_{1}$ \\
\hline Rule-Based & 6.6 & 43.8 & 61.3 & 39.5 & 40.4 \\
Copy Net & 11.0 & 37.0 & 28.1 & $\mathbf{5 0 . 1}$ & 28.4 \\
Attn. Seq2Seq & 10.2 & 30.0 & 30.0 & 42.4 & 17.9 \\
KV Retrieval Net (no enc. attn.) & 10.8 & 40.9 & 59.5 & 35.6 & 36.6 \\
KV Retrieval Net & $\mathbf{1 3 . 2}$ & $\mathbf{4 8 . 0}$ & $\mathbf{6 2 . 9}$ & 47.0 & $\mathbf{4 1 . 3}$ \\
\hline Human Performance & 13.5 & 60.7 & 64.3 & 61.6 & 55.2 \\
\hline
\end{tabular}

Table 3: Evaluation on our test data. Bold values indicate best model performance. We provide both an aggregated $F_{1}$ score as well as domain-specific $F_{1}$ scores. Attn. Seq2Seq refers to a sequence-tosequence model with encoder attention. KV Retrieval Net (no enc. attn.) refers to our new model with no encoder attention context vector computed during decoding.

model performed worse on our data with this added mechanism. We choose this model for comparison as it is also end-to-end trainable and implicitly models dialogue state through learned neural representations, putting it in the same class of dialogue models as our key-value retrieval net. This model has also been shown to be a competitive task-oriented dialogue baseline that can accurately interpret user input and act on this input through latent distributed representation. We refer to this model as Copy Net in the results tables.

\subsection{Automatic Evaluation}

\subsubsection{Metrics}

Though prior work has shown that automatic evaluation metrics often correlate poorly with human assessments of dialogue agents (Liu et al., 2016), we report a number of automatic metrics in Table 3. These metrics are provided for coarse-grained evaluation of dialogue response quality:

- BLEU: We use the BLEU metric, commonly employed in evaluating machine translation systems (Papineni et al., 2002), which has also been used in past literature for evaluating dialogue systems both of the chatbot and task-oriented variety (Ritter et al., 2011; Li et al., 2016; Wen et al., 2016b). While work by (Liu et al., 2016) has demonstrated that ngram based evaluation metrics such as BLEU and METEOR do not correlate well with human performance on non-task-oriented dialogue datasets, recently (Sharma et al., 2017) have shown that these metrics can show comparatively stronger correlation with human assessment on task-oriented datasets. We, therefore, calculate average BLEU score over all responses generated by the system, and primarily report these scores to gauge our model's ability to accurately generate the language patterns seen in our data.

- Entity $\mathbf{F}_{1}$ : Each human Turker's Car Assistant response in the test data defines a gold set of entities. To compute an entity $F_{1}$, we micro-average over the entire set of system dialogue responses and use the entities in their canonicalized forms. This metric evaluates the model's ability to generate relevant entities from the underlying knowledge base and to capture the semantics of the userinitiated dialogue flow. Given that our test set contains dialogues from all three domains, we compute a per-domain entity $F_{1}$ as well as an aggregated dataset entity $F_{1}$. We note that other work on task-oriented dialogue by (Wen et al., 2016b; Henderson et al., 2014a) have reported the slot-tracking accuracy of their systems, which is a similar but perhaps more informative and fine-grained notion of a system's ability to capture user semantics. Because our model does not have provisions for slot-tracking by design, we are unable to report such a metric and hence report our entity $F_{1}$.

\subsubsection{Results}

We see that of our baseline models, Copy Net has the lowest aggregate entity $F_{1}$ performance. Though it has the highest model entity $F_{1}$ for the weather domain dialogues, it performs very poorly in the other domains, indicating its inability to generalize well to multiple dialogue domains and to accurately integrate relevant entities into its responses. Copy Net does, however, have the second highest BLEU score, which is not surprising given that the model is a powerful extension to the sequence-to-sequence modelling class, which is known to have very robust language modelling capabilities. 
Our rule-based model has the lowest BLEU score, which is a consequence of the fact that the naturalness of the system output is very limited by the number of diverse and distinct response templates we manually provided. This is a common issue with heuristic dialogue agents and one that could be partially alleviated through a larger collection of lexically rich response templates. However, the rule-based system has a very competitive aggregate entity $F_{1}$. This is because it was designed to accurately parse the semantics of user utterances and query the underlying $\mathrm{KB}$ of the dialogue, through manually-provided heuristics.

As precursors to our key-value retrieval net, we first report results of a model that does not compute an attention over the KB (referred to as Attn. Seq2Seq) and show that without computing attention over the $\mathrm{KB}$, the model performs poorly in entity $F_{1}$ as its output is agnostic to the world state represented in the KB. Note that this model is effectively a sequence-to-sequence model with encoder attention. If we include an attention over the KB but do not compute an encoder attention (referred to as KV Retrieval Net no enc. attn.), the entity $F_{1}$ increases drastically, showing that the model is able to incorporate relevant entities from the KB. Finally, we combine these two attention mechanisms to get our final key-value retrieval net. Our proposed key-value retrieval net has the highest modelling performance in BLEU, aggregate entity $F_{1}$, and entity $F_{1}$ for the scheduling and navigation domains. It outperforms the rule-based aggregate entity $\mathrm{F}_{1}$ by $4.2 \%$ and outperforms the Copy Net BLEU score by 2.2 points as well as its entity $F_{1}$ by $11 \%$. These salient gains are noteworthy because our model is able to achieve them by learning its latent representationts directly from data, without the need for heuristics or manual labelling.

We also report human performance on the provided metrics. These scores were computed by taking the dialogues of the test set and having a second distinct batch of Amazon Mechanical Turk workers provide system responses given prior dialogue context. This, in effect, functions as an interannotator agreement score and sets a human upper bound on model performance. We see that there is a sizable gap between human performance on entity $\mathrm{F}_{1}$ and that of our key-value retrieval net ( $\sim 12.7 \%$ ), though our model is on par with human performance in BLEU score.

\subsection{Human Evaluation}

We randomly generated 120 distinct scenarios across the three dialogue domains, where a scenario is defined by an underlying $\mathrm{KB}$ as well as a user goal for the dialogue (e.g. find the nearest gas station, avoiding heavy traffic). We then paired Amazon Mechanical Turkers with one of our systems in a real-time chat environment, where each Turker played the role of the Driver. We evaluated the rule-based model, Copy Net, and key-value retrieval network on each of the 120 scenarios. We also paired a Turker with another Turker for each of the scenarios, in order to get evaluations of human performance. At the end of the chat, the Turker was asked to judge the quality of their partner according to fluency, cooperativeness, and humanlikeness on a scale from 1 to 5 . The average scores per pairing are reported in Table 4. In a separate experiment, we also had Turkers evaluate the outputs of the systems on 80 randomly selected dialogues from the test split of our dataset. Those outputs were evaluated according to correctness, appropriateness, and humanlikeness of the responses, and the scores are reported in Table 5 .

We see that on real-time dialogues the key-value retrieval network outperforms the baseline models on all of the metrics, with especially sizeable performance gains over the Copy Net which is the only other recurrent neural model evaluated. We also see that human performance on this assessment sets the upper bound on scores, as expected. The results on human evaluation of test outputs show that the rule-based model provides the most correct system responses, the KV network provides the most appropriate responses, and the Copy Net gives the most humanlike responses by small margins. We should note, however, that the second regime for human evaluation is more unrealistic because it involves providing a dialogue context that is directly sampled from our dataset, whereas the first regime of real-time dialogues measures the models' abilities to adapt to new and noisier user input. This suggests that the first set of results are more meaningful and representative for assessing overall model efficacy.

Examples of dialogues conducted between our model and Turkers are included in Figure 3. Particularly noteworthy is our model's ability to seamlessly integrate world information from the underlying KBs in the respective dialogues, while 


\begin{tabular}{l|ccc} 
Model & Fluency & Cooperative & Humanlike \\
\hline Rule-based & 3.20 & 3.39 & 2.87 \\
Copy Net & 2.33 & 2.42 & 2.02 \\
KV Ret. Net & $\mathbf{3 . 3 6}$ & $\mathbf{3 . 4 3}$ & $\mathbf{3 . 0 9}$ \\
\hline Human & 3.98 & 3.95 & 4.04 \\
\hline
\end{tabular}

Table 4: Human evaluation results on realtime dialogues.

still producing very naturalistic utterances. The model is able to do this effectively across multiple domains.

\begin{tabular}{|c|c|c|c|}
\hline POI & Category & Traffic Info & $\ldots$ \\
\hline Civic Center & parking garage & car collision & $\ldots$ \\
Valero & gas station & road block & $\ldots$ \\
Webster Garage & parking garage & car collision & $\ldots$ \\
Trader Joes & grocery store & heavy & $\ldots$ \\
Mandarin Rts. & chinese rest. & moderate & $\ldots$ \\
$\ldots$ & $\ldots$ & $\ldots$ & $\ldots$ \\
\hline
\end{tabular}

DRIVER: I am looking for a gas station near me with the shortest route from me.

CAR: the closest gas station is valero but, but there is a road block nearby.

DRIVER: What is the next nearest gas station?

CAR: $\quad$ valero is the only gas station that $i$ can find

DRIVER: Thanks

CAR: here to serve.

\begin{tabular}{|c|c|c|c|c|}
\hline Event & Time & Date & Party & Agenda \\
\hline swimming act. & $3 \mathrm{pm}$ & the 11th & sister & - \\
dinner & $7 \mathrm{pm}$ & the 3rd & mother & - \\
football & $2 \mathrm{pm}$ & the 20 th & mother & - \\
lab appt. & $10 \mathrm{am}$ & the 17 th & Jeff & - \\
$\ldots$ & $\ldots$ & $\ldots$ & $\ldots$ & $\ldots$ \\
\hline
\end{tabular}

DRIVER: car when is gonna be my next swimming

$\begin{array}{ll}\text { CAR: } & \begin{array}{l}\text { activity? } \\ \text { at } 3 p m \text { next swimming activity is on the 11th }\end{array} \\ \text { DRIVER: } & \text { who is gonna attend with me? } \\ \text { CAR: } & \text { your swimming activity is on the 11th, one at } \\ & \text { 3pm with sister } \\ \text { DRIVER: } & \text { thank you! } \\ \text { CAR: } & \text { you are welcome }\end{array}$

Figure 3: Sample dialogues from our human evaluation, conducted between a Turker and the KV Retrieval Net. In the dialogue, our model responses are italicized. Additional dialogues are included in the supplementary material.

\section{Conclusion and Future Work}

In this work, we have presented a novel neural task-oriented dialogue model that is able to sustain grounded discourse across a variety of domains by retrieving world knowledge represented in knowledge bases. It smoothly incorporates

\begin{tabular}{l|ccc} 
Model & Correct & Appropriate & Humanlike \\
\hline Rule-based & $\mathbf{3 . 9 6}$ & 3.57 & 3.28 \\
Copy Net & 3.52 & 3.63 & $\mathbf{3 . 5 6}$ \\
KV Ret. Net & 3.70 & $\mathbf{3 . 6 4}$ & 3.50 \\
\hline
\end{tabular}

Table 5: Human evaluation of system outputs on test set.

this world knowledge into natural-sounding system responses in an end-to-end trainable fashion, without the need to explicitly model dialogue state. Our model outperforms competitive heuristic and neural baselines on both automatic and human evaluation metrics. In addition, we have introduced a publicly available dialogue dataset across three domains in the in-car personal assistant space that we hope will help the data scarcity issue present in task-oriented dialogue research.

Future work will address closing the margin between the Key-Value Retrieval Network and human performance on the various metrics. This will include developing new methods for robust handling of joint $\mathrm{KB}$ attributes as well as usage of the $\mathrm{KB}$ that requires more pragmatic understanding of the world via notions such as temporal reasoning.

\section{Acknowledgments}

The authors wish to thank He He, Peng Qi, Urvashi Khandelwal, and Reid Pryzant for their valuable feedback and insights. We gratefully acknowledge the funding of the Ford Research and Innovation Center, under Grant No. 124344.

\section{References}

L. El Asri, H. Schulz, S. Sharma, J. Zumer, J. Harris, E. Fine, R. Mehrotra, and K. Suleman. 2017. Frames: A corpus for adding memory to goal-oriented dialogue systems. http://www.maluuba.com/publications/ .

Dzimitry Bahdanau, Kyunghyun Cho, and Yoshua Bengio. 2015. Neural machine translation by jointly learning to align and translate. In International Conference on Learning Representations (ICLR2015).

Christina Bennett and Alexander I. Rudnicky. 2002. The carnegie mellon communicator corpus. In $I C$ SLP.

Antoine Bordes and Jason Weston. 2016. Learning end-to-end goal-oriented dialog. arXiv preprint arXiv:1605.07683

Bhuwan Dhingra, Lihong Li, Xiujun Li, Jianfeng Gao, Yun-Nung Chen, Faisal Ahmed, and Li Deng. 
2016. End-to-end reinforcement learning of dialogue agents for information access. arXiv preprint arXiv:1609.00777.

Mihail Eric and Christopher Manning. 2017. A copyaugmented sequence-to-sequence architecture gives good performance on task-oriented dialogue. In Proceedings of the 15th Conference of the European Chapter of the Association for Computational Linguistics: Volume 2, Short Papers. Association for Computational Linguistics, pages 468-473. http://aclweb.org/anthology/E17-2075.

Jiatao $\mathrm{Gu}$, Zhengdong Lu, Hang Li, and Victor O.K. Li. 2016. Incorporating copying mechanism in sequence-to-sequence learning. In Proceedings of the 54th Annual Meeting of the Association for Computational Linguistics (Volume 1: Long Papers). Association for Computational Linguistics, Berlin, Germany, pages 1631-1640. http://www.aclweb.org/anthology/P16-1154.

Caglar Gulcehre, Sungjin Ahn, Ramesh Nallapati, Bowen Zhou, and Yoshua Bengio. 2016. Pointing the unknown words. In Proceedings of the 54th Annual Meeting of the Association for Computational Linguistics (Volume 1: Long Papers). Association for Computational Linguistics, Berlin, Germany, pages 140-149. http://www.aclweb.org/anthology/P16-1014.

C.T. Hemphill, J. J. Godfrey, and G. R. Doddington. 1990. The atis spoken language systems pilot corpus. In Proceedings of the DARPA speech and natural language workshop.

Matthew Henderson, Blaise Thomson, and Jason Williams. 2014a. The second dialog state tracking challenge. 15th Annual Meeting of the Special Interest Group on Discourse and Dialogue page 263.

Matthew Henderson, Blaise Thomson, and Steve Young. 2014b. Word-based dialog state tracking with recurrent neural networks. In Proceedings of the SIGDIAL 2014 Conference.

Geoffrey E. Hinton, Nitish Srivastava, Alex Krizhevsky, Ilya Sutskever, and Ruslan R. Salakhutdinov. 2012. Improving neural networks by preventing co-adaptation of feature detectors. arXiv preprint arXiv:1207.0580 .

Sepp Hochreiter and Jurgen Schmidhuber. 1997. Long short-term memory. Neural Computation pages 1735-1780.

Robin Jia and Percy Liang. 2016. Data recombination for neural semantic parsing. In Proceedings of the 54th Annual Meeting of the Association for Computational Linguistics (Volume 1: Long Papers). Association for Computational Linguistics, Berlin, Germany, pages 12-22. http://www.aclweb.org/anthology/P16-1002.
Anjuli Kannan, Karol Kurach, Sujith Ravi, Tobias Kaufman, Balint Miklos, Greg Corrado, Andrew Tomkins, Laszlo Lukacs, Marina Ganea, Peter Young, and Vivek Ramavajjala. 2016. Smart reply: Automated response suggestion for email. In Proceedings of the ACM SIGKDD Conference on Knowledge Discovery and Data Mining (KDD) (2016).. https://arxiv.org/pdf/1606.04870v1.pdf.

Diederik Kingma and Jimmy Ba. 2015. Adam: a method for stochastic optimization. In International Conference on Learning Representations (ICLR2015).

Jiwei Li, Michel Galley, Chris Brockett, Jianfeng Gao, and Bill Dolan. 2016. A diversity-promoting objective function for neural conversation models. In Proceedings of the 2016 Conference of the North American Chapter of the Association for Computational Linguistics: Human Language Technologies. Association for Computational Linguistics, San Diego, California, pages 110-119. http://www.aclweb.org/anthology/N16-1014.

Wang Ling, Phil Blunsom, Edward Grefenstette, Karl Moritz Hermann, Tomáš Kočiský, Fumin Wang, and Andrew Senior. 2016. Latent predictor networks for code generation. In Proceedings of the 54th Annual Meeting of the Association for Computational Linguistics (Volume 1: Long Papers). Association for Computational Linguistics, Berlin, Germany, pages 599-609. http://www.aclweb.org/anthology/P16-1057.

Chia-Wei Liu, Ryan Lowe, Iulian Serban, Mike Noseworthy, Laurent Charlin, and Joelle Pineau. 2016. How not to evaluate your dialogue system: An empirical study of unsupervised evaluation metrics for dialogue response generation. In Proceedings of the 2016 Conference on Empirical Methods in Natural Language Processing. Association for Computational Linguistics, Austin, Texas, pages 2122-2132. https://aclweb.org/anthology/D16-1230.

Fei Liu and Julien Perez. 2016. Gated endto-end memory networks. arXiv preprint arXiv:1610.04211.

Minh-Thang Luong, Hieu Pham, and Christopher D. Manning. 2015a. Effective approaches to attentionbased neural machine translation. Empirical Methods in Natural Language Processing pages 14121421.

Alexander Miller, Adam Fisch, Jesse Dodge, AmirHossein Karimi, Antoine Bordes, and Jason Weston. 2016. Key-value memory networks for directly reading documents. In Proceedings of the 2016 Conference on Empirical Methods in Natural Language Processing. Association for Computational Linguistics, Austin, Texas, pages 1400-1409. https://aclweb.org/anthology/D16-1147.

Kishore Papineni, Salim Roukos, Todd Ward, and Wei-Jing Zhu. 2002. Bleu: a method 
for automatic evaluation of machine translation. In Proceedings of 40th Annual Meeting of the Association for Computational Linguistics. Association for Computational Linguistics, Philadelphia, Pennsylvania, USA, pages 311-318. https://doi.org/10.3115/1073083.1073135.

Vu Pham, Theodore Bluche, Christopher Kermorvant, and Jerome Louradour. 2014. Dropout improves recurrent neural networks for handwriting recognition. arXiv preprint arXiv: $1312.4569 v 2$.

Alan Ritter, Colin Cherry, and William B. Dolan. 2011. Data-driven response generation in social media. Empirical Methods in Natural Language Processing pages 583-593.

Sikhar Sharma, Layla El Asri, Hannes Schulz, and Jeremie Zumer. 2017. Relevance of unsupervised metrics in task-oriented dialogue for evaluating natural language generation. arXiv preprint arXiv:1706.09799.

David Sussillo and L.F. Abbott. 2015. Random walk initialization for training very deep feed forward networks. arXiv preprint arXiv:1412.6558 .

Ilya Sutskever, Oriol Vinyals, and Quoc V Le. 2014. Sequence to sequence learning with neural networks. In Z. Ghahramani, M. Welling, C. Cortes, N. D. Lawrence, and K. Q. Weinberger, editors, Advances in Neural Information Processing Systems 27, Curran Associates, Inc., pages 3104 3112. http://papers.nips.cc/paper/5346-sequenceto-sequence-learning-with-neural-networks.pdf.

Oriol Vinyals, Ł ukasz Kaiser, Terry Koo, Slav Petrov, Ilya Sutskever, and Geoffrey Hinton. 2015. Grammar as a foreign language. In C. Cortes, N. D. Lawrence, D. D. Lee, M. Sugiyama, and R. Garnett, editors, Advances in Neural Information Processing Systems 28, Curran Associates, Inc., pages 27732781. http://papers.nips.cc/paper/5635-grammaras-a-foreign-language.pdf.

Tsung-Hsien Wen, David Vandyke, Milica Gasic, Nikola Mrksic, Lina. M. Rojas-Barahona, Pei-Hao $\mathrm{Su}$, Stefan Ultes, and Steve Young. 2016b. A network-based end-to-end trainable task-oriented dialogue system. arXiv preprint arXiv:1604.04562 .

Jason D. Williams, Kavosh Asadi, and Geoffrey Zweig. 2017. Hybrid code networks: practical and efficient end-to-end dialog control with supervised and reinforcement learning. arXiv preprint arXiv:1702.03274.

Jason D. Williams, Antoine Raux, Deepak Ramachadran, and Alan Black. 2013. The dialog state tracking challenge. In Proceedings of the SIGDIAL. Metz, France.

Steve Young, Milica Gasic, Blaise Thomson, and Jason D. Williams. 2013. POMDP-based statistical spoken dialog systems: a review. Proceedings of the IEEE 28(1):114-133. 


\begin{tabular}{|c|c|c|c|c|}
\hline POI & Category & Address & Traffic Info & Distance \\
\hline Dish Parking & parking garage & 550 Alester Ave & no traffic & 3 miles \\
Cafe Venetia & coffee or tea place & 329 El Camino Real & road block nearby & 4 miles \\
Mandarin Roots & chinese restaurant & 271 Springer St & car collision nearby & 3 miles \\
Dominos & pizza restaurant & 776 Arastradero Rd & moderate traffic & 6 miles \\
Travelers Lodge & rest stop & 333 Arbol Dr & moderate traffic & 4 miles \\
\hline
\end{tabular}

DRIVER: What is the nearest parking garage within 4 miles

that has no traffic

CAR: $\quad$ the nearest is dish parking at 550 alester ave, 3 miles away

DRIVER: Does it have any traffic?

CAR: there is no traffic on the route to dish parking

DRIVER: Thanks

CAR: you're welcome!

\begin{tabular}{|c|c|c|c|c|c|}
\hline Event & Time & Date & Party & Agenda & Location \\
\hline dinner & $5 \mathrm{pm}$ & the 4th & brother & - & - \\
meeting & $1 \mathrm{pm}$ & the 18 th & vice president & discuss company picnic & room 100 \\
conference & $11 \mathrm{am}$ & the $1 \mathrm{st}$ & HR & go over budget & room 50 \\
meeting & $4 \mathrm{pm}$ & the 9 th & HR & onboard new members & room 100 \\
lab appt. & $6 \mathrm{pm}$ & the 14 th & Jon & - & - \\
dinner & $6 \mathrm{pm}$ & the $1 \mathrm{st}$ & Jon & - & - \\
\hline
\end{tabular}

DRIVER: When is my dinner going to be and who will be attending

CAR: $\quad y o u r$ next dinner is on the 1 st at $6 \mathrm{pm}$ with your jon

DRIVER: Great, thanks.

CAR: you're welcome!

\begin{tabular}{|c|c|c|c|c|}
\hline POI & Category & Address & Traffic Info & Distance \\
\hline Dominos & pizza restaurant & 776 Arastradero Rd & moderate traffic & 7 miles \\
Stanford Express Care & hospital & 214 El Camino Real & car collision nearby & 1 miles \\
Travelers Lodge & rest stop & 333 Arbol Dr & no traffic & 4 miles \\
Coupa & coffee or tea place & 394 Van Ness Ave & moderate traffic & 7 miles \\
The Clement Hotel & rest stop & 657 Ames Ave & road block nearby & 6 miles \\
5672 barringer st. & certain address & 5672 barringer st. & car collision nearby & 4 miles \\
\hline
\end{tabular}

DRIVER: Hi, I'd like to find a coffee place near me

CAR: we're 7 miles away from coupa but there is a moderate traffic

DRIVER: What's the address?

CAR: $\quad$ the address is 394 van ness ave, i sent it on your screen

DRIVER: Great. Can you find me the fastest route there?

CAR: the fastest route is for coupa.

DRIVER: Ok, so coupa is the closest place and you have the fastest route there

CAR: the fastest route has moderate traffic and there is a moderate traffic on the route

DRIVER: yes

CAR: $\quad i$ sent the info on your screen, drive carefully!

DRIVER: Thanks

CAR: $\quad$ you're welcome, have a great day.

\begin{tabular}{|l|c|c|c|c|c|c|}
\hline & monday & tuesday & wednesday & thursday & friday & $\ldots$ \\
\hline los angeles & hail, 60-70f & foggy, 30-40f & cloudy, 20-30f & stormy 30-40f & drizzle, 30-50f & $\ldots$ \\
durham & rain, 80-90f & cloudy, 50-70f & misty, 40-60f & dry, 80-100f & rain, 50-70f & $\ldots$ \\
boston & overcast, 30-50f & misty, 40-60f & foggy, 40-50f & foggy, 40-50f & clear skies 20-30f & $\ldots$ \\
seattle & warm, 60-70f & foggy, 30-40f & cloudy, 20-30f & stormy, 30-40f & drizzle, 30-50f & $\ldots$ \\
fresno & clear skies, 40-60f & drizzle, 70-80f & humid, 70-90f & stormy, 60-70f & cloudy, 90-100f & $\ldots$ \\
san francisco & foggy, 40-60f & hot, 90-100f & humid, 50-70f & rain, 80-100f & misty, 20-40f & $\ldots$ \\
alhambra & dew, 30-40f & dry, 30-50f & dry, 50-60f & humid, 80-90f & snow, 60-80f & $\ldots$ \\
\hline
\end{tabular}

DRIVER: how is the weather

CAR: $\quad$ what city would you like the weather for?

DRIVER: is it foggy in seattle?

CAR: $\quad$ it will not be foggy in seattle today

DRIVER: thanks

CAR: $\quad$ you're welcome!

Figure 4: Sample dialogues from our model. Note in the weather dialogue, the current day is italicized. 
Figure 5: An image provided to users to elicit unbiased audio commands for prompting more naturalistic dialogues
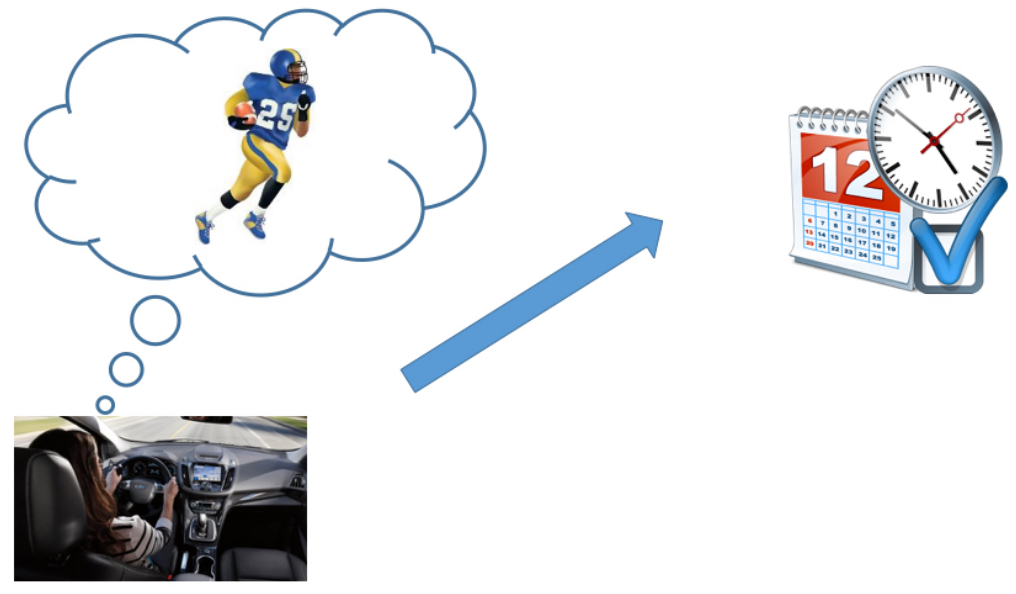

Figure 6: Driver mode in the wizard-of-oz collection scheme

TASK: You want to know the forecast in new york for the following period of time: today and tomorrow

DRIVER response:

tell me the temperature in new york]

Figure 7: Car Assistant mode in the wizard-of-oz collection scheme

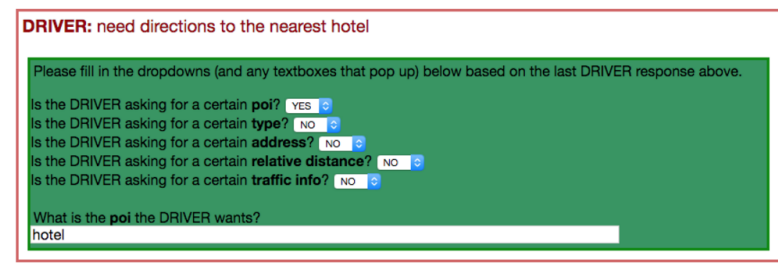

\begin{tabular}{|c|c|c|c|c|}
\multicolumn{5}{c|}{ Location Information } \\
\hline relative distance & traffic info & address & type & poi \\
\hline 5 miles & no traffic & 465 Arcadia Pl & rest stop & Four Seasons \\
\hline 3 miles & no traffic & 550 Alester Ave & parking garage & Dish Parking \\
\hline \hline 6 miles & moderate traffic & 347 Alta Mesa Ave & rriends house & jills house \\
\hline 5 miles & no traffic & 5677 springer street & certain address & 5677 springer street \\
\hline \hline 5 miles & no traffic & 638 Amherst St & grocery store & Sigona Farmers Market \\
\hline
\end{tabular}

Now, fill in what you as the CAR ASSISTANT would say to the DRIVER below

CAR ASSISTANT response:

End of dialogue? ONLY click this if the last DRIVER statement above suggests the task is done $->$ 